Nouvelles perspectives en sciences sociales

\title{
Conférence inaugurale du colloque de Sudbury
}

La revue Nouvelles perspectives en sciences sociales et la sociologie contemporaine. Un programme de refondation

\section{Claude Vautier}

Volume 11, numéro 2, mai 2016

Sur le thème : complexité et relation

URI : https://id.erudit.org/iderudit/1037101ar

DOI : https://doi.org/10.7202/1037101ar

Aller au sommaire du numéro

Éditeur(s)

Prise de parole

ISSN

1712-8307 (imprimé)

1918-7475 (numérique)

Découvrir la revue

Citer ce document

Vautier, C. (2016). Conférence inaugurale du colloque de Sudbury : la revue Nouvelles perspectives en sciences sociales et la sociologie contemporaine. Un programme de refondation. Nouvelles perspectives en sciences sociales, 11(2), 23-41. https://doi.org/10.7202/1037101ar d'utilisation que vous pouvez consulter en ligne. 


\section{Conférence inaugurale du colloque de Sudbury}

\section{La revue Nouvelles perspectives en sciences sociales et la sociologie contemporaine. Un programme de refondation}

Claude Vautier

Rédacteur en chef de la revue NPSS

\section{Les raisons du colloque}

T e colloque qui s'est tenu à Sudbury, les 7 et 8 mai 2015, est né de la volonté de célébrer un anniversaire, celui de la revue Nouvelles perspectives en sciences sociales (NPSS). Ce colloque vise à faire un point d'étape et à dessiner la contribution que ses dirigeants entendent qu'elle apporte à la sociologie contemporaine.

Créée dans une période où ses fondateurs percevaient des signes d'épuisement d'une sociologie passée depuis plus de vingt ans sous la coupe de l'individualisme méthodologique, NPSS ${ }^{1}$ avait pour vocation de proposer des voies de recherche alternatives ou, au moins, complémentaires.

$\overline{1}$ Le titre de cette revue correspond, à lui seul, à un programme invitant les chercheurs en sciences humaines et sociales à dessiner de nouvelles perspectives, c'est-à-dire de nouvelles approches méthodologiques dans le double cadre de la " pensée complexe » et des " études relationnelles". 
Je souhaite montrer qu'elle a atteint un seuil où de nouveaux choix s'imposent et proposer certains de ces choix pour l'avenir.

Je le ferai en évoquant successivement les deux points suivants :

- la genèse de la revue : petite histoire de la création d'une revue (1),

- l'état de son parcours scientifique et sa place dans les SHS contemporaines (2).

\section{Genèse et histoire de la revue}

Le premier volume de NPSS est paru en mai 2005. En ce mois de mai 2015, non seulement NPSS est toujours publiée, mais encore elle a pris sa place dans le paysage éditorial des SHS. Je vais évoquer cette évolution en cinq étapes.

1.1. Les origines de NPSS : conception

Lidée de créer cette revue est née quelque part entre Sudbury, Toulouse et un petit village de la côte sud de la Crète, en 2002. C'est là, au cours d'un bel après-midi d'automne (on était en octobre), sur une plage méditerranéenne crétoise, ciel bleu, mer bleue, que, d'échanges de propos en émissions d'idées, se dessinait ce qui devint un programme de travail à réaliser. Simon Laflamme était au stylo et constituait l'une de ces listes dont il a le secret et, surtout, la capacité à les épuiser jusqu'au dernier signe. Dans cette liste, un projet paraissant particulièrement ambitieux ou peu raisonnable : créer une nouvelle revue de sociologie ou de SHS.

L'idée couvait sans doute des deux côtés de l'Atlantique. Du côté de Toulouse, un petit groupe de chercheurs vivement intéressés par la "pensée complexe » d'Edgar Morin. Côté canadien, un autre petit groupe de chercheurs très orientés vers les « études relationnelles ".

Les deux groupes avaient beaucoup à partager et à apprendre l'un de l'autre.

Les Toulousains étaient centrés sur le projet d'opérationnaliser la pensée de Morin qu'avait proposé celui qui dirigeait alors le 
CIRESS $^{2}$, Pascal Roggero. Nous cherchions donc à construire des modèles qui respectent les grandes caractéristiques de la pensée complexe : récursivité organisationnelle, dialogie, hologrammie, et quelques autres injonctions, telles conjoindre, et non disjoindre, intégrer le temps...

À Sudbury, autour de Simon Laflamme, c'est une approche relationnelle qui se constituait. On y était également conscient des impératifs de la pensée complexe, mais c’était là, sans doute, la " moindre des choses ", tandis que la conception d'Edgar Morin était abordée sous d'autres angles. Ce qui était le plus important selon les canadiens, pour pouvoir appliquer la pensée complexe, c'était de sortir des modélisations centrées sur l'acteur et sa psyché. Simon Laflamme fustigeait ainsi toutes les sociologies phénoménologiques, psychologisantes, interactionnistes qui dominaient la scène sociologique... Il nous reprochait même, avec bienveillance mais clairement, à nous qui avions décidé de nouer des liens avec lui, de n'être pas sortis de cette posture de façon claire.

Bien entendu, de part et d'autre, on se sentait un peu seul, puisque la planète sociologie était très largement colonisée par ces courants qui remontaient aux débuts de la discipline et étaient aussi peu propices à la mise en œuvre d'une pensée complexe que d'une pensée relationnelle.

C'est d'ailleurs l'une des raisons qui ont conduit à la création de NPSS : le sentiment que certains des refus d'articles proposés aux principales revues académiques provenaient du fait que l'on avait du mal à accepter, voire à comprendre, nos orientations, nous renvoyant fréquemment à la lecture des auteurs eux-mêmes académiques dont il semblait, selon nos détracteurs, que nous étions ignorants.

\subsection{La création de NPSS : naissance et enfance}

Nous avons donc créé une revue de systémique complexe et d'études relationnelles.

$\overline{2}$ Centre Interdisciplinaire de Recherche et d'Études sur les Systèmes Sociaux, disparu depuis. 
À Sudbury, Simon Laflamme et Ali Reguigui se sont mis en ordre de bataille.

Prise de parole, éditeur situé à Sudbury (Ontario) pressenti pour assurer l'édition a accepté le défi.

Ali Reguigui, professeur de linguistique à l'Université Laurentienne, est devenu le rédacteur en chef de la revue dont la maquette a été créée dans cette même université et dont le site a été hébergé également par la Laurentienne, jusqu'en 2013.

Le comité de rédaction se limitait à 3 personnes, pour les deux premiers numéros, puis à 4 lorsque j'ai rejoint ce comité, à partir du volume $2, \mathrm{n}^{\circ} 1$ (septembre 2006). On m’a demandé alors d'être rédacteur en chef, ce qui est devenu effectif pour le numéro suivant (volume $2, \mathrm{n}^{\circ} 2$, mars 2007).

Les premiers numéros ont été construits avec les moyens du bord, c'est-à-dire en exploitant nos réseaux les plus proches. Les deux groupes ont été utilisés au mieux. Nous avions, les uns et les autres, des choses à dire et nous les avons écrites. Nous avons écumé une bonne part des possibilités « locales ", je veux dire des amis et collègues les plus proches. Mais, dès le volume $2, \mathrm{n}^{\circ} 2$, nous pouvions publier des auteurs plus éloignés : la revue restait fragile, certes, mais elle était lancée. Le comité de rédaction compte aujourd'hui 14 membres et, depuis le volume $4, \mathrm{n}^{\circ} 2$ (avril 2009), les numéros sont tous thématiques. Premier thème : "La complexité ».

Les volumes successifs qui nous ont conduit au volume 10, $\mathrm{n}^{\circ} 2$, que Benoît Feildel, l'un de ses co-directeurs et membre du comité de rédaction, va nous présenter en fin de journée, ont développé des thèmes tels que "La relation comme débat fondamental de la sociologie contemporaine " (volume $5, \mathrm{n}^{\circ} 1$, octobre 2009), « La simulation dans les sciences humaines » (volume 5 , $\mathrm{n}^{\circ}$ 2, mai 2010), "La minorité linguistique " (volume 6, $\mathrm{n}^{\circ} 1$, décembre 2010), «Les économistes dans la cité » (volume 6, ${ }^{\circ} 2$, juin 2011, "Y a-t-il une théorie en interdisciplinarité ?" (volume $7, \mathrm{n}^{\circ} 1$, octobre 2011), «La modélisation " (volume 7 , $\mathrm{n}^{\circ}$ 2, mai 2012), "L'homogénéisation et la différenciation " (volume $8, \mathrm{n}^{\circ} 1$, novembre 2012), «Représentations identitaires 
et expressions culturelles de la francophonie canadienne à travers ses pratiques artistiques et médiatiques " (volume $8, \mathrm{n}^{\circ} 2$, mai 2013), "Linguistique et complexité " (volume 9, $\mathrm{n}^{\circ} 1$, novembre 2013), "L'échange » (volume 9, $\mathrm{n}^{\circ} 2$, mai 2014), "Le territoire " (volume 10, $\mathrm{n}^{\circ} 1$, novembre 2014) et enfin, "Les temporalités en SHS » (volume 10, $\mathrm{n}^{\circ}$ 2, avril 2015). On remarque que les thèmes sont variés, avec des appartenances à la sociologie, la linguistique, l'économie, l'urbanisme et aménagement ou encore l'épistémologie et l'analyses de données textuelles informatisée ${ }^{3}$.

Ces thèmes qui ont réuni des spécialistes connus dont les analyses n'étaient pas forcément partagées par les membres du comité de rédaction, ont tous à voir avec l'objectif fixé, dès le départ à la revue, par ses fondateur et que l'on trouve depuis le premier numéro, dans son énoncé de mission. Il s'agit, pour la revue, d'accueillir les pensées scientifiques novatrices et rompant avec les modélisations centrées sur la psyché des acteurs, tout en restant ouverte à une pluralité d'approches : "L'ouverture à toute pensée novatrice et rigoureuse doit être une règle, car il est peu probable que les territoires qui se révèlent livrent à l'observateur autre chose que la pluralité aussi bien des objets d'analyse que de la manière de les aborder ${ }^{4}$ ". Cette pluralité correspond à une volonté qui est d'essayer de faire dialoguer les pensées entre elles, fussent-elles contradictoires.

\subsection{Les années d'adolescence}

Jusqu'au volume $5, \mathrm{n}^{\circ} 1$, chaque ouvrage publié comportait environ 150 pages et alignait en moyenne entre 4 et 5 articles.

Désormais, chaque volume comporte entre 250 et 350 pages et propose entre 7 et 10 articles $^{5}$. Bien entendu, le nombre de pages, pas plus que le nombre d'articles par numéro ne sont des indicateurs de qualité des publications. Par contre ils sont des

3 Au moment de la publication de cette conférence, NPSS s'est enrichie du volume $11, \mathrm{n}^{\circ} 1,2015$, sur ce dernier thème.

Énoncé de mission, p. 1.

Pour le volume $11, \mathrm{n}^{\circ} 1$, c'est 481 pages, 11 articles et 7 compte-rendus de lecture. 
indices de l'essor de la revue. Car dans le même temps, d'une part, du fait des appels à contribution qui sont désormais systématiquement publiés sur notre site, nous recevons des articles sans avoir besoin de les solliciter individuellement, comme c'était le cas au début. D'autre part, nous avons sept volumes en cours qui doivent paraître entre novembre 2015 et novembre 2018, leur logistique est en place, avec un directeur, un appel à contribution est en préparation ou déjà sur le site et nous disposons de textes susceptibles d'être publiés en varia dans ces volumes.

J'ai parlé métaphoriquement d'adolescence de la revue pour dire deux choses : elle a évolué normalement vers une autonomie et une régularité de fonctionnement; elle reste cependant un peu en deçà de ce que l'on peut lire dans son énoncé de mission : la revue a pour objectif d'ouvrir ses pages à des chercheurs et à des travaux scientifiques critiques à l'égard des théories dominantes en SHS, particulièrement se situant " en dehors d'une logique phénoménologique où l'essentiel s'explique par référence aux intentions des acteurs ${ }^{6} »$.

Son évolution n'est donc pas achevée.

Au tout début, il fallait d'abord que la revue s'installe dans le paysage éditorial des SHS, au moins en France et au Canada, et même dans diverses autres régions ou pays francophones. Elle devait, pour cela, accueillir des travaux de qualité, même s'ils étaient éloignés de sa propre ligne scientifique. C'est ce qui a été fait.

Il ne s'agissait pas simplement de "realpolitik» : non seulement l'énoncé de mission ouvre la revue à diverses traditions, mais encore, puisque nous sommes dans une position critique (pas seulement, nous essayons aussi de construire une autre sociologie), nous devons donner la parole à ceux dont nous critiquons les approches, les travaux, les résultats. Cela signifie que NPSS continuera à publier des textes écrits par des auteurs dont nous reconnaissons les qualités, mais qui sont éloignés ou opposés à nos propres manières de penser.

$6 \quad$ Énoncé de mission, p. 2. 


\subsection{L'âge adulte}

NPSS est aujourd'hui une revue connue et appréciée dans les milieux de la recherche en SHS. Bien entendu, ce n'est pas la Revue française de sociologie fondée en 1960 par Jean Stoetzel, ni L'Année sociologique fondée par Durkheim en 1898 et toujours publiée, bien que dans une autre optique ou encore les Actes de la recherche en sciences sociales créés par Pierre Bourdieu en 1975.

Les deux dernières revues que je viens de citer ont été fondées clairement dans le but de soutenir une pensée, un mode de pensée, pour faire école. C'est particulièrement net pour L'Année sociologique de Durkheim qui fut l'instrument du durkheimisme avant la première guerre mondiale, puis de l'individualisme méthodologique, lorsque Raymond Boudon devint président de son comité de rédaction, entre 1978 et 2013, année de sa mort.

On retrouve ces traits, sous une forme différente, dans les Actes : Bourdieu, qui en est le rédacteur en chef, ne signe pas d'éditoriaux pour affirmer la ligne de la revue, mais, depuis 1975 et jusqu'à sa disparition en 2000, il écrit, dans chaque numéro, un ou plusieurs articles où se trouve défendue une position consistant à " [sélectionner] la production d'un vaste réseau international de chercheurs de sciences humaines et sociales, dans la perspective d'une sociologie critique des modes de domination ${ }^{7} \|^{8}$.

Entrer dans l'âge adulte, pour notre revue, consiste à affirmer son propre caractère.

Tout en accueillant des auteurs divers provenant d'horizons divers, NPSS doit aussi participer à la promotion d'un mode de pensée formant contraste avec les approches les plus répandues.

$\overline{7} \quad$ Cette phrase figure sur le site des archives des Actes (depuis 1975) présent sur le portail Persée.

8 Quant à la Revue française de sociologie, la présentation qu'on en trouve sur le site Persée, où ses archives sont mises en ligne, est plus neutre : "la Revue française de sociologie publie des travaux qui contribuent à la connaissance du monde social et au développement des outils, conceptuels et techniques, de cette connaissance ». On se souvient, cependant que Jean Stoetzel fut le directeur de thèse de Raymond Boudon, lequel fut membre du comité de rédaction de la revue entre 1965 et 1990. Mais Jean-Claude Chamboredon (1967-1990), Edgar Morin (1960-1971), par exemple, firent aussi partie de ce comité... 
Elle doit donc faire une place aux auteurs qui souhaitent explorer d'autres paradigmes et tenter l'expérience relationnelle. Cela signifie aussi que ces derniers doivent proposer des articles à la revue, et je pense ici particulièrement à ceux qui en sont les plus proches, notamment ceux qui jouent un rôle important dans son comité de rédaction.

À titre personnel, j'ai longtemps souhaité que les auteurs publiant dans NPSS ne soient pas trop fréquemment les membres de son comité de rédaction. Ma crainte était que ne se développe une sorte d'autoproduction et un risque de consanguinité... Au contraire, il me semble que nous avons désormais intérêt à faire vivre nos pages d'un souffle qui anime le débat, la controverse, qui permette de mettre en œuvre une véritable proposition alternative.

Il serait aujourd'hui paradoxal que, disposant d'une revue qui a réussi à s'installer dans le paysage éditorial, les tenants des approches relationnelles et de la systémique complexe laissent cet instrument dormir.

Nous savons les résultats obtenus par les courants cités précédemment, en partie grâce aux revues qui leur servirent de tremplin.

NPSS peut être un tremplin similaire pour la pensée complexe relationnelle.

1.5. Le coût et le goût de l'autonomie

NPSS est soutenue par Prise de parole qui l'édite depuis son premier numéro. Grâce à Simon Laflamme, Ali Reguigui et quelques autres, l'Université Laurentienne nous apporte également un soutien par le biais, notamment, de la mise à disposition partielle d'une secrétaire, Carole G. Anderson. Le LEREPS, petit laboratoire toulousain aux moyens très limités, soutient le seul sociologue qu'il compte encore dans ses rangs comme il le peut, et moralement quand il ne peut faire plus.

Mais ce sont là les seuls appuis dont nous disposons. Notre demande de référencement auprès de l'AERES (Agence d'Évaluation de la Recherche et de l'Enseignement Supérieur) déposée 
en novembre 2013, n'a toujours pas reçu de réponse. Nous restons donc en attente, tout en considérant que la consultation des listes de revues agréées par l'AERES ne semble pas attester que NPSS y dénoterait.

Quelques mots supplémentaires, notamment pour les Canadiens et tous ceux qui ne vivent pas au sein de l'institution universitaire française. Le référencement par l'AERES et, désormais, le HCERES, permet aux chercheurs français de nourrir leur dossier personnel de carrière. Les articles publiés dans des revues non référencées ne sont simplement pas comptabilisés lors de l'évaluation du chercheur. Ainsi, les jeunes enseignants-chercheurs et les doctorants qui publient chez nous ne se voient-ils pas crédités des textes que nous avons publiés.

Nous pourrions nous passer du référencement par le HCERES, mais c'est aussi l'une des missions d'une revue que d'offrir des opportunités de publication à ceux qui débutent dans ce difficile et passionnant métier.

Nous pourrions d'ailleurs également, sous la réserve précédente, nous en passer dans la mesure où notre revue est présente dans des index tels que Sociological Abstracts ou Social Sciences Citation Index qui convergent vers le portail ProQuest; distribuée par Erudit.org, NPSS jouit donc d'une visibilité importante dans le monde francophone, malgré les flottements constatés en France même.

\section{La place de NPSS dans le paysage des SHS contemporaines}

Mais si l'autonomie a un coût, elle a aussi le goût de la liberté, notamment de la liberté de penser autrement. Et nous avons souhaité, depuis sa création, que cette revue dispose d'un ancrage particulier dans la sociologie et les SHS contemporaines.

C'est l'un des points importants que je veux souligner en ouverture de ce colloque.

2.1. La synthèse du doyen Davy

Le premier numéro de la Revue française de sociologie, par la plume de Georges Davy qui fut l'élève de Durkheim (élève 
imposé, mais qui devint un fervent disciple du maître), évoque le risque d'une sociologie réduisant l'homme à sa seule dimension sociale, l'amputant d'une part de lui-même :

il apparait que si l'explication sociologique devait imposer plus que la priorité, mais le monopole aussi, du facteur social, comme si tout individuel signifiait arbitraire et danger de naufrage scientifique, elle réduirait, sans vraie raison, l'homme à sa seule dimension sociale. Elle l'amputerait d'une part de lui-même : de cette singularité propre où il se croit parfois le droit de se reconnaitre...?

Seize ans plus tard, dans l'article nécrologique qu'il consacre à Davy, Jean Stoetzel rappelle en même temps une formule de ce dernier datant de 1911 : « la sociologie est donc vraiment et plus complètement que la psychologie une science de l'homme». Et il ajoute, évoquant la querelle entre les psychologues et les sociologues des années 1930 : " il apparaissait que Durkheim et ses amis avaient choisi leur bord. On voit qu'Essertier choisissait l'autre. Or, ce que ce dernier ne percevait pas, c'est que Davy était déjà sur la voie de la synthèse entre psychologie et sociologie ${ }^{10}$ ".

Nous pouvons considérer que la "synthèse " recherchée par Georges Davy n'est pas la voie qu'a emprunté la sociologie de la seconde moitié du $\mathrm{XX}^{\mathrm{e}}$ siècle. Pour la caractériser à grands traits, on peut admettre qu'en suivant Davy, elle aurait eu pour projet de développer une science humaine et sociale dans laquelle l'humain n'est pas tout tandis que la société n'intervient également que de façon partielle.

Mais, dans les années 1960, Raymond Boudon se prépare à imposer l'individualisme méthodologique en sociologie (il existe depuis deux siècles en économie), et ce courant, qui sera si bien en phase avec la "science économique » des années 1970, va déferler sur la sociologie et imposer une vision de la société dans laquelle la synthèse de Davy n'a, provisoirement, plus d'espace.

9 Georges Davy, "Émile Durkheim ", Revue française de sociologie, vol. 1, $\mathrm{n}^{\mathrm{o}}$ 1, 1960, p. 24, http://www.persee.fr/web/revues/home/prescript/article/ rfsoc_0035-2969_1960_num_1_1_1733, site consulté le 5 avril 2015. Jean Stoetzel, "Georges Davy, 1883-1976 ", Revue française de sociologie, vol. 17, no 2, 1976, p. 161-162. 
La sociologie des années 1980-2010 environ est une sociologie qui se réclame résolument de l'agent, de l'acteur, de l'individu, de l'homme, en tant qu'atome de la vie sociale. Ce sont ses caractéristiques d'humain, son intentionnalité, sa rationalité, ses valeurs, ses capacités cognitives, qui lui permettent d'agir et, ainsi, de créer la société, par les interactions qu'il a avec les autres agents, acteurs, etc. D'une certaine façon, ce qu'il reste du projet de Davy, c'est le côté psychologique. Mais, la psychologie est réduite à des capacités caricaturales et la société se trouve réduite à un ensemble d'interactions même pas socialisées ni socialisantes. L'acteur de l'individualisme méthodologique est, quoi qu'ait pu en dire Raymond Boudon, un imbécile partiellement rationnel, et autiste de surcroît, puisque ses interactions ne changent rien à ses décisions. On a beau peaufiner sa rationalité de principe, voire limiter cette rationalité, y mettre du manque d'information, des émotions susceptibles de brouiller ses cartes ${ }^{11} \ldots$ rien n'y fait. Les modèles sociologiques, comme les modèles économiques, ne peuvent rendre compte d'une réalité qu'ils appauvrissent beaucoup trop.

Ce que nous voulions avec la création de NPSS, c'était sortir de cette impasse qui nous contraignait, soit à croire et/ou propager l'idée que la lutte des classes expliquait l'évolution historique des nations ou que les positions sociales des individus étaient essentiellement déterminées par la distribution des positions sociales de leurs parents, qu'il n'y avait donc pas de possibilité d'évolution d'une structure sociale; soit à penser et/ou écrire que l'individu est forcément responsable de son destin puisque ses

11 Le concept d'émorationalité, inventée, à ma connaissance, par Simon Laflamme (voir Communication et émotion. Essai de microsociologie relationnelle, Paris, L'Harmattan, 1995), peut être utilisé au moins dans deux directions : il peut être considéré comme une amélioration du concept de rationalité et servir dans des modélisations basées sur les jeux d'acteurs; mais il peut également être utilisé en arrière-fond pour comprendre ce qui revient à l'humain, dans une modélisation relationnelle trinitaire où l'individu est l'une des catégories agissantes aux côtés du système et du temps. De ce point de vue, et contrairement à certaines critiques selon lesquelles l'approche relationnelle ne serait, au final, qu'un avatar du holisme, l'individu prend toute sa place - mais aussi rien que sa place - dans les modélisations relationnelles. 
choix rationnels lui appartiennent et que, sauf ou malgré les effet pervers, les individus, qui ont toujours de bonnes raisons de faire ce qu'ils font, parviennent à imposer leurs choix dans les systèmes sociétaux.

L'objectif de cette deuxième partie de la conférence, c'est de montrer, bien que le terme puisse paraître prétentieux, que c'est d'une tentative de refondation qu'il s'agit. NPSS trace des lignes de recherche, propose des orientations et des résultats capables d'apporter de l'intelligibilité et du sens à une multitude de questions que les théories dominantes ne font qu'effleurer.

Je vais essayer de décrire ces lignes de force en les illustrant avec trois des thématiques proposées aux auteurs depuis le volume 4, no 2 en 2009.

\subsection{Complexité et relation}

Ces deux termes sont à la base de notre travail de refondation. C'est pourquoi nous les avons proposés pour thèmes des deux premiers numéros thématiques.

Le premier de ces deux termes, désormais entré dans un vocabulaire commun, a eu tendance à se dégrader, à s'affadir au fur et à mesure qu'il s'imposait dans le langage vernaculaire et même dans celui, a priori clairement spécifié, des scientifiques. Nous sommes amenés à constater chaque jour que des auteurs utilisent le terme complexité sans aucune tentative de définition, sans aucune référence à ceux qui ont dessiné des contours à ce concept. Or, le terme "complexité " est loin d'être fixé, clarifié. Il est au contraire polysémique, comporte des zones d'ombre, des plages floues, des incertitudes, des orientations discutées et sans doute discutables. Les six textes du volume $4, \mathrm{n}^{\circ} 2$, sur ce thème ("La complexité ») ne pouvaient pas épuiser la question, mais étaient en mesure de nous éveiller à un débat salutaire sur les multiples dimensions et acceptions du concept. Et de nous convaincre de la nécessité d'interroger sans cesse les définitions possibles, souhaitables, pertinentes... de ce terme.

Le terme "relation " est moins prégnant dans la langue des SHS. Il est cependant, lui aussi, sujet à des interprétations 
divergentes : entre la relation telle que la conçoit Simon Laflamme et la manière dont les adeptes de la théorie des réseaux la voient, il y a un écart important. Pour l'un, la relation est fondatrice, première, elle est le postulat de base : les individus sont toujours déjà en relation, en dehors de leur volonté même. Pour les autres, c'est parce que les acteurs décident de se mettre en relation qu'apparaît un réseau. Malgré des proximités indéniables entre les deux approches, la coupure est nette sur ce point central.

On peut considérer que la relation est associée aux « comportements réactionnels ${ }^{12}$ " dont parle Weber et au fait que comprendre une activité significative ne puisse se faire que "sous la forme d'un comportement d'une ou plusieurs personnes singulières $^{13} "$.

Mais on peut aussi, comme le fait Laflamme, écrire que : "L'approche relationnelle à laquelle nous souscrivons met aussi l'accent sur le lien plutôt que sur les objets qui sont reliés [...] L'acteur social est actif dans le champ des relations; mais son action procède de relations ${ }^{14}$ ".

\subsection{Complexité relationnelle}

NPSS est une revue de "systémique complexe et d'études relationnelles".

La juxtaposition qui apparaît grâce au " et » de cet intitulé me semble gênante aujourd'hui. Pour moi, elle est au moins tautologique. Dans mon esprit, la systémique complexe se devrait d'être relationnelle, ce qu'elle n'est pas forcément. Par contre, pour ce qui est de l'approche relationnelle, on pourrait montrer qu'elle est complexe, par nature, pourrait-on dire. Sur ces deux points, je renvoie au moins à Morin 1977 - Méthode, $1^{15}$.

12 Max Weber, Économie et société, 1, Les catégories de la sociologie, Paris, Plon, coll. " Pocket », 1995 [posthume, 1921], p. 29.

13 Ibid., p. 40.

14 Simon Laflamme, "Sciences sociales et approche relationnelle ", Nouvelles perspectives en sciences sociales, volume 5, $\mathrm{n}^{\circ} 1,2009$, p. 84.

15 Edgar Morin, La Méthode, 1, Paris, Seuil, 1977, p. 123-24, "Au-delà du holisme et du réductionnisme : le circuit relationnel " : " ni la description ni l'explication d'un système ne peuvent s'effectuer au niveau des parties, conçues comme entités isolées, liées seulement par actions et réactions ». 
Personnellement, je préférerais maintenant dire que NPSS est une revue de systémique complexe relationnelle, ce qui mêle intimement les deux approches, au lieu de simplement les articuler.

Cette proposition n'est pas sans conséquence. Les travaux actuels qui s'inscrivent dans une perspective de systémique complexe se divisent clairement en deux groupes inégaux. Pour les auteurs les plus nombreux, les systèmes complexes, avec leurs caractéristiques définies notamment par Edgar Morin et JeanLouis Le Moigne, apparaissent comme issus des interactions entre individus, agents, acteurs... ${ }^{16,17}$.

Les modélisations mises en œuvre (je renvoie à NPSS, volume 7, $\mathrm{n}^{\circ} 2^{18}$ ) sont donc susceptibles d'être classées en deux grandes branches.

Soit elles mettent en scène des acteurs que l'on essaie de rendre complexes, en leur accordant des psychés moins élémentaires, de la rationalité, certes, mais aussi de l'émotion, en les plaçant dans des systèmes eux-mêmes complexifiés par l'existence en leur sein de contradictions ou paradoxes (principe dialogique), de récursivité organisationnelle, d'hologrammie, de projectivité, et en soumettant l'ensemble acteurs-système à des conditions non aristotéliciennes, c'est-à-dire s'inscrivant contre les axiomes d'identité, de noncontradiction et de tiers exclu...

De telles approches, si elles améliorent l'appréhension des phénomènes sociétaux, souffrent cependant de la nécessité persistante de faire des hypothèses sur les raisons des choix et des actions des individus. Ce sont ces hypothèses qui constituent la gangue de l'analyse et en remettent en question la validité au moins partiellement.

Soit elles peuvent également mettre en scène, non des interactions entre des individus, mais des relations entre des catégories ana-

16 C'est le cas, il est vrai plutôt singulier, des études fondées sur des SMA (Systèmes Multi-Agents), tels qu'ils sont présentés dans le volume 5, n 2 de la revue, mai 2010.

17 Les autres considèrent qu'un système peut s'étudier sans faire appel aux acteurs et, soit en restent à une cybernétique finalement peu complexe, soit pratiquent un holisme non exempt de certains défauts du holisme méthodologique.

Sur le thème de la modélisation, mai 2012. 
lytiques. C'est ce qu'a fait Simon Laflamme en mettant en relation les personnes, les idées et les biens et en étudiant comment s'effectuait la circulation de chaque catégorie dans le champ des deux autres, plus précisément de la relation entre les deux autres.

Un tel modèle est bien moins incarné. Il est bien moins intuitif. Il ne nous fournit pas de représentation évidente ou " réaliste » de ce qu'est une société. Pourtant, les êtres humains y sont, leurs idées et représentations également; les biens au sens économique du terme sont également dans la boucle et circulent, s'échangent, le système vit avec ses normes, ses règles, sa re(trait d'union) production, comme disait Barel...

Contrairement à ce que suggèrent des critiques qui pensent que l'individu n'existe plus dans cette sociologie qui serait une sorte de théorie durkheimienne désincarnée du tout sociétal, l'individu est bien présent dans le système qui est un système d'échange généralisé : de façon symbolique, des individus se partagent ou échangent des idées et des biens, mais des biens et des idées se partagent des personnes, des idées, des biens... Cela, c'est vrai, devient sérieusement abstrait!

Et, cependant, cette abstraction me semble bien moins perturbante pour l'analyse que celle d'un homo sociologicus, même particulièrement peaufiné et subtil, qui sera toujours, qu'on me permette l'expression, "à côté de la plaque ".

D'autant qu'un autre élément important doit être impulsé dans la manière de faire de la sociologie de nos jours : le temps. La plupart des modèles sociologiques sont statiques. Lorsqu'ils introduisent le temps, c'est sous la forme d'une statique comparative : deux situations séparées dans le temps sont étudiées, chacune de façon statique, et le rapprochement des résultats au temps 1 et au temps 2 permet de parler de changement.

Cependant, rien n'apparaît quant au processus qui est à l'œuvre et qui a permis le passage de l'état 1 à l'état 2. Le volume $10, \mathrm{n}^{\circ} 2$ qui vient d'être publié traite de cette question, en très grande partie dans l'optique particulière des territoires et des politiques publiques ${ }^{19}$.

$\overline{19}$ Sur le thème des temporalités, avril 2015. 
Nous devons généraliser la prise en compte du temps et je crois que c'est par des formes différentes de modélisation des systèmes complexes que nous pourrons y arriver.

\section{Conclusion - Pour une sociologie relationnelle : modéliser des systèmes complexes relationnels ou modéliser relationnellement des systèmes complexes}

Il n'existe pas, à ma connaissance de revue de systémique complexe telle que NPSS.

Il n'existe pas, et ici je suis sûr de moi, de revue de systémique complexe et d'études relationnelles, mis à part notre revue.

Mais il n'existe pas non plus de revue de systémique complexe relationnelle.

Jusqu'à présent, nous avons essayé de rassembler les deux approches qui étaient celles des deux groupes de chercheurs qui se sont réunis pour créer NPSS : les uns faisaient de la systémique complexe, les autres des études relationnelles.

À la longue, il m'est apparu que si le groupe pratiquant les études relationnelles pratiquait aussi, de facto, la systémique complexe, le groupe spécialisé dans cette dernière, ne connaissait pas l'approche relationnelle, ou, en tout cas, ne la pratiquait pas.

Il est vrai que la manière de pratiquer la systémique complexe n'était pas la même de part et d'autre de l'Atlantique. Mais la pratique reposait cependant sur les mêmes concepts, les mêmes auteurs : Edgar Morin et sa Méthode était le socle incontesté dans les deux cas.

Quelques différences apparaissaient pourtant ici et là. Laflamme trouvait que le propos de Morin avait tendance à favoriser une vision interactionniste de la société. Et de fait, les "systémiciens " avaient bien tendance à modéliser dans le sens d'un interactionnisme, mesuré, mais dans lequel la psychologie de convention jouait un rôle non négligeable.

Le constat reste valable. Les travaux actuels autour de NPSS gardent quelque chose de cette partition entre des modélisations à base d'acteurs et des modélisations à base relationnelle. 
Mon sentiment personnel, mais je sais qu'il est partagé par plusieurs d'entre nous, est que nous devons sortir de cette ambiguïté.

Il ne s'agit pas d'imposer une pratique au détriment d'une autre, mais de bien spécifier ce que chacune d'elle a de particulier. À mon avis, il faut aussi essayer de montrer qu'une approche relationnelle clairement assumée est susceptible de nous faire sortir des impasses dans lesquelles nous nous trouvons ${ }^{20}$.

Cela signifie que nous devrions développer, non plus une systémique complexe et des études relationnelles, mais une systémique complexe relationnelle à laquelle devrait être associée une modélisation des systèmes complexes relationnels ou, mieux, me semble-t-il, une modélisation relationnelle des systèmes complexes.

Délaissant les théories de l'action, de l'intention, de la rationalité, de l'interaction et, principalement aujourd'hui, l'individualisme méthodologique, nous devons aller sans détour vers une théorie de la relation :

- dans laquelle individu, système et temps (avec son cortège d'imprévisibilités) sont indissociablement liés;

- dans laquelle aucune d'entre ces trois catégories analytiques (qui sont toutes bien plus riches que leur intitulé ne semble le dire) n'est le moteur de la construction de la société;

- dans laquelle, loin des hypothèses psychologiques absurdes et irréalistes, des informations construites à partir d'histoires de vie, par exemple, des données caractérisant les systèmes étudiés et les événements capables de transformer une situation par leur survenue dans le cours du temps, permettent de dresser une représentation à la fois sensée et objectivée du monde que nous tentons de comprendre et expliquer.

$\overline{20}$ Qui se traduisent notamment par un éclatement et l'orientation vers autant de sociologies qu'il y a d'objets à étudier : sociologie du genre, sociologie du sport, des transports, de la santé, du travail, des organisations... 
Personne n'est obligé de suivre cette voie et les deux façons de pratiquer la systémique complexe ont droit de cité.

À titre personnel, je me suis engagé dans la perspective relationnelle qui me paraît aller plus loin et de façon plus prometteuse. C'est la raison pour laquelle j'invite à assigner à NPSS l'objectif de promouvoir le paradigme relationnel et d'aider à développer des travaux de systémique complexe relationnelle et des modélisations relationnelles des systèmes complexes durant les années futures.

Pour ce faire, j’invite également chacun à participer à ce projet.

Je propose, notamment, que se tienne désormais, de façon régulière (ce pourrait être tous les deux ou trois ans), un colloque NPSS destiné à favoriser et évaluer l'avancement de ce projet.

Je vous remercie de votre attention. 


\section{Quelques éléments bibliographiques}

Davy, Georges, "Émile Durkheim ", Revue française de sociologie, vol. 1, $\mathrm{n}^{\circ}$ 1, 1960, p. 24, http://www.persee.fr/web/revues/home/prescript/article/ rfsoc_0035-2969_1960_num_1_1_1733, site consulté le 5 avril 2015.

Laflamme, Simon, Communication et émotion. Essai de microsociologie relationnelle, Paris, L'Harmattan, 1995

Weber, Max, Économie et société, 1, Les catégories de la sociologie, Paris, Plon, coll. " Pocket », 1995 [posthume, 1921].

Laflamme, Simon, "Sciences sociales et approche relationnelle ", Nouvelles perspectives en sciences sociales, vol. $5, \mathrm{n}^{\circ} 1, \mathrm{p} .79-85$.

Morin, Edgar, La Méthode, 1, Paris, Seuil, 1977.

Stoetzel, Jean, "Georges Davy, 1883-1976 ", Revue française de sociologie, vol. 17, $\mathrm{n}^{\circ} 2,1976$.

Weber, Max, Économie et société, 1. Les catégories de la sociologie, Paris, Plon, coll. " Pocket », 1995 [posthume, 1921].

Tous les numéros de NPSS évoqués sont répertoriés sur le site de la revue et consultables (sous la réserve de la barrière mobile de deux années) sur Erudit.org. 